\title{
Downregulated exosome-associated gene FGF9 as a novel diagnostic and prognostic target for ovarian cancer and its underlying roles in immune regulation
}

\author{
Zhijie $\mathrm{Xu}^{1,2,3,{ }^{*}}$, Yuan $\mathrm{Cai}^{1{ }^{1,}}$, Wei Liu4, Fanhua Kang${ }^{2}$, Qingchun He ${ }^{5,6}$, Qianhui Hong ${ }^{2}$, Wenqin \\ Zhang ${ }^{2}$, Jianbo $\mathrm{Li}^{2}$, Yuanliang Yan ${ }^{7}$, Jinwu Peng ${ }^{1,2}$ \\ ${ }^{1}$ Department of Pathology, Xiangya Hospital, Central South University, Changsha 410008, Hunan, China \\ ${ }^{2}$ Department of Pathology, Xiangya Changde Hospital, Changde 415000, Hunan, China \\ ${ }^{3}$ National Clinical Research Center for Geriatric Disorders, Xiangya Hospital, Central South University, Changsha \\ 410008, Hunan, China \\ ${ }^{4}$ Department of Orthopedic Surgery, The Second Hospital University of South China, Hengyang 421001, Hunan, \\ China \\ ${ }^{5}$ Department of Emergency, Xiangya Hospital, Central South University, Changsha 410008, Hunan, China \\ ${ }^{6}$ Department of Emergency, Xiangya Changde Hospital, Changde 415000, Hunan, China \\ ${ }^{7}$ Department of Pharmacy, Xiangya Hospital, Central South University, Changsha 410008, Hunan, China \\ *Equal contribution
}

Correspondence to: Yuanliang Yan, Jinwu Peng; email: yanyuanliang@csu.edu.cn, jinwupeng@csu.edu.cn

Keywords: ovarian cancer, exosome, FGF9, prognosis, immune regulation

Received: January 7, $2022 \quad$ Accepted: February 15, $2022 \quad$ Published: February 21, 2022

Copyright: (C) $2022 \mathrm{Xu}$ et al. This is an open access article distributed under the terms of the Creative Commons Attribution License (CC BY 3.0), which permits unrestricted use, distribution, and reproduction in any medium, provided the original author and source are credited.

\begin{abstract}
Exosome has been demonstrated to be secreted from cells and seized by targeted cells. Exosome could transmit signals and exert biological functions in cancer progression. Nevertheless, the underlying mechanisms of exosome in ovarian cancer (OC) have not been fully explored. In this study, we wanted to explore whether Fibroblast growth factor 9 (FGF9), as an exosome-associated gene, was importantly essential in OC progression and prognosis. Firstly, comprehensive bioinformatics platforms were applied to find that FGF9 expression was lower in OC tissues compared to normal ovarian tissues. Meanwhile, downregulated FGF9 displayed favorable prognostic values in OC patients. The gene enrichment of biological functions indicated that abnormally expressed FGF9 could be involved in the OC-related immune signatures, such as immunoinhibitors and chemokine receptors. Taken together, these findings could provide a novel insight into the significance of FGF 9 in OC progress and supply a new destination of FGF9-related immunotherapy in clinical treatment.
\end{abstract}

\section{INTRODUCTION}

The incidence rate of ovarian cancer ranks the third place all over the world. And of all the malignant tumors, the fatality rate of ovarian cancer is the highest [1]. In recent days, numerous kinds of clinical treatments have been applied to the patients with ovarian cancer, including surgery, chemotherapy. Whereas, for patients that are diagnosed in the late stage, the 5-year survival rate accounts for $<25 \%$. And the effects of conventional treatments are not as good as before [2]. Therefore, it is significantly important to explore a novel biomarker and new clinical therapies to improve the overall rate and prognosis of ovarian cancer patients.

Exploring the mechanisms of tumor immune microenvironment (TIME) could be beneficial for the 
guidance of the immune responses of cancer and predict the therapeutic molecules [3, 4]. To have a better understanding of the TIME in ovarian cancer is necessary. Because immune cells could enhance anticancer activities and decrease the recurrence rate of cancer. Immune systems combined with signaling biomarkers could play a crucial role in the prognostic prediction of ovarian cancer patients [5]. Nevertheless, more profound scientific researches regarding the relationships between TIME and ovarian cancer are still essential and needed to be fully investigated.

Exosomes were demonstrated to be normal nanovesicles which were composed of various molecules, including lipid and nucleic acids. They were found in the exocytosis of cells and could contribute to the biological functions of cells, which could serve as a biomarker in the pathological process of human diseases and cancers [6-8]. Exosomes had a strong relationship with immune responses in numerous diseases, such as cardiovascular, central nervous system and cancer. Furthermore, the utility of exosome-related immune regulation could do good to the future therapeutic progression [9, 10]. Fibroblast growth factor 9 (FGF9), as an exosomeassociated gene, was first found in human glioma cells, and it has been reported to participate in the regulation in glia of central nervous system [11]. Recently, numerous studies have demonstrated that FGF9 plays a crucial part in the tumor progression. The upregulated level of FGF9 exerted great effects in the transdifferentiation of small cell lung cancer (SCLC). In vivo studies have identified that FGF9 induced the malignant transformation via triggering FGFR pathway [12]. Moreover, abnormally expressed FGF9 was involved in the modulation of OC invasiveness [13]. Whereas, more studies are still required to investigate the relationship between FGF9 expression and the prognosis of OC patients.

In this paper, we would explore the underlying mechanisms of FGF9 in ovarian cancer. Through comprehensive bioinformatic analysis, the expression level of FGF9 was discovered to be downregulated in ovarian cancer tissues. And the high expression of FGF9 has strong correlation with good prognosis. These findings indicated that FGF9 could be a novel prognostic prediction and immune-associated biomarker for ovarian cancer.

\section{MATERIALS AND METHODS}

\section{Data acquisition}

Gene Expression Omnibus (GEO) database [14] was applied to explore and download the two OC datasets, including GSE26712 [15, 16], GSE18520 [17] (Table 1).
Then, we have analyzed the differently expressed genes (DEGs) between the normal ovarian tissues and OC tissues. The cut-off value was established: $p$-value $<0.05$ and $|\log F C| \geq 2.0$. In order to explore the codifferentially expressed genes (co-DEGs) of the exosome-associated gene dataset and two GEO datasets, the Venn plots were employed. Moreover, the Cancer Genome Atlas (TCGA) database [18] was used to obtain the expression levels and clinical statistics of OC patients.

\section{Bioinformatics platforms}

The comprehensive evaluations of differently expressed genes were downloaded from some bioinformatic platforms (Table 2). The prognostic values were evaluated by means of the Kaplan-Meier plotter [19]. This database was used for the exploration of the overall survival (OS), first-progression survival (FPS) and post progression survival (PPS) of co-DEGs in OC. Additionally, the TNMplot [20], TCGA database and GEPIA2.0 [21] further investigated the expression level of FGF9 in tumor group and normal group. Subsequently, the LinkedOmics platform [22] was used to analyze the correlation between FGF9 and coexpressed genes. At the same time, through this database, we have identified the Gene Ontology (GO) and Kyoto Encyclopedia of Genes and Genomes (KEGG) pathways. Concerning the research of the link between FGF9 and immune response and regulation, we employed the single-sample GSEA (ssGSEA), TISIDB [23] and TIMER [24]. Subsequently, the relationships between FGF9 expression and immune checkpoints, including CTLA4 and VSIR have been figured out.

\section{Statistical analysis}

The findings in this study were depicted as mean \pm standard deviation (SD). The difference between the normal group and the tumor group was investigated by $t$-test. $P<0.05$ was regarded to be statistically significant.

\section{RESULTS}

\section{Differently expressed genes between ovarian cancer group and normal group}

Through the exploration in GEO database, we finally picked out two suitable datasets and then attained the statistics of the two datasets. The cut-off value was set up: $p$-value $<0.05$ and $|\operatorname{logFC}| \geq 2.0$. After this procedure, DEGs between ovarian cancer group and normal group were generated. And we found that in GSE26712, there were 82 genes up-regulated and 231 genes down-regulated. Meanwhile, there were 493 
Table 1. The features of two GEO datasets about gene expression profiling by array.

\begin{tabular}{lcccccc}
\hline \multirow{2}{*}{ GEO datasets } & \multirow{2}{*}{ Platform } & \multicolumn{2}{c}{ Sample size } & DEGs & References \\
\cline { 3 - 4 } & & cancer & normal & & \\
\hline GSE26712 & GPL96 & 185 & 10 & 82 up-regulated genes and 231 down-regulated genes & {$[15,16]$} \\
GSE18520 & GPL570 & 53 & 10 & & 493 up-regulated genes and 599 down-regulated genes & {$[17]$} \\
\hline
\end{tabular}

Abbreviations: GEO: Gene Expression Omnibus datasets; DEGs: differentially expressed genes.

Table 2. Bioinformatics platforms that are employed to analyze the role of FGF9 in ovarian cancer.

\begin{tabular}{lcc}
\hline Database & URL & References \\
\hline GEO & $\underline{\text { https://www.ncbi.nlm.nih.gov/gds/?term= }}$ & {$[14]$} \\
TCGA & $\underline{\text { https://portal.gdc.cancer.gov/ }}$ & {$[18]$} \\
Kaplan-Meier Plotter & $\underline{\mathrm{http}: / / \text { mmplot.com/analysis/ }}$ & {$[19]$} \\
TNMplot & $\underline{\mathrm{http} / / \text { www.tnmplot.com }}$ & {$[20]$} \\
GEPIA2.0 & $\underline{\text { http://gepia.cancer-pku.cn/ }}$ & {$[21]$} \\
LinkedOmics & $\underline{\mathrm{http} / / \text { www.linkedomics.org/admin.php }}$ & {$[22]$} \\
TISIDB & $\underline{\mathrm{http} / / / \text { cis.hku.hk/TISIDB/ }}$ & {$[23]$} \\
TIMER & $\underline{\text { https://cistrome.shinyapps.io/timer/ }}$ & {$[24]$} \\
\hline
\end{tabular}

up-regulated genes and 599 down-regulated genes in GSE18520 (Supplementary Table 1). Besides, we applied the Venn plot (http://bioinformatics.psb. ugent.be/webtools/Venn/) to implicate the significance of exosome-correlated genes in the development of OC patients. And the Venn plot showed that two upregulated exosome-correlated genes (CD24 and CP) and one downregulated exosome-correlated gene (FGF9) might play pivotal roles in OC progression (Figure 1).

\section{Up-regulation}

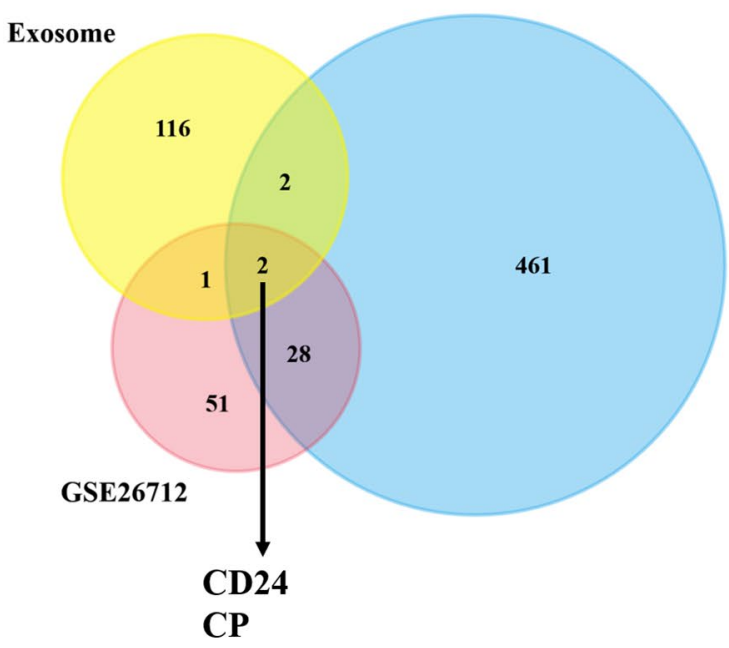

The prognostic prediction value of FGF9 in ovarian cancer patients

To investigate the correlation between the expression of CD24, CP, FGF9 and the OC patients' prognosis, the Kaplan-Meier plotter was applied. The findings have conveyed that high expression level of CD24 was linked to good OS (HR $=0.87,95 \% \mathrm{CI}=0.76-0.98, p=$ $0.028)$, PFS ( $\mathrm{HR}=0.86,95 \% \mathrm{CI}=0.75-0.99, p=0.04)$, PPS $(\mathrm{HR}=0.82,95 \% \mathrm{CI}=0.69-0.97, p=0.022)$

\section{Down-regulation}

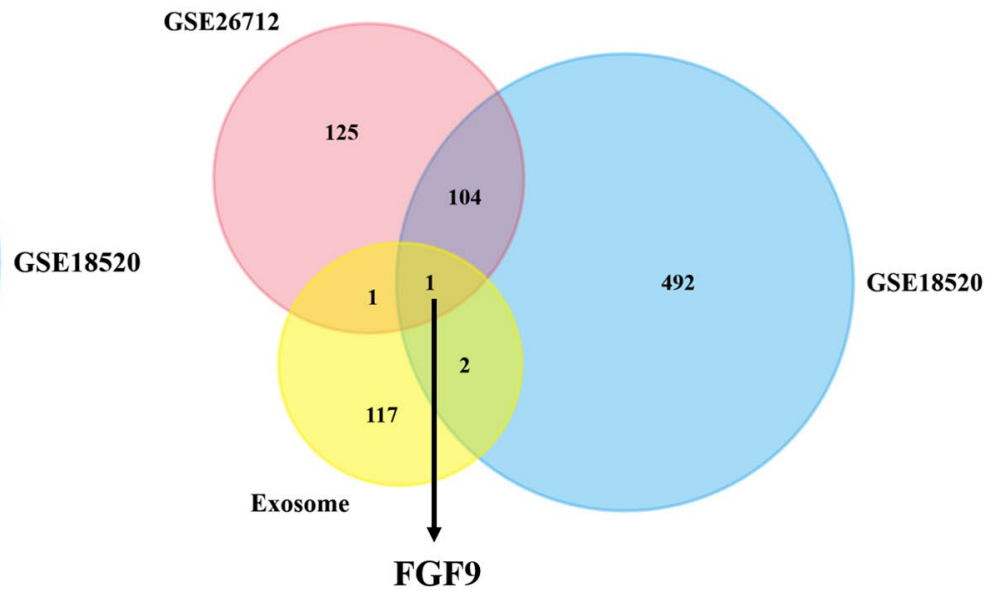

Figure 1. The co-DEGs between the exosome-associated genes and two OC datasets. The Venn plot showed that two upregulated exosome-correlated genes (CD24 and CP) and one downregulated exosome-correlated gene (FGF9) might play pivotal roles in OC progression. 
(Figure 2A-2C). Furthermore, high expression of CP was related to favorable $\mathrm{OS}$ ( $\mathrm{HR}=0.79,95 \% \mathrm{CI}=$ $0.63-0.99, p=0.043)$, PPS (HR $=0.73,95 \% \mathrm{CI}=0.55$ $0.95, p=0.02$ ) (Figure 2D, 2F). However, CP expression was not correlated with the PFS of OC patients $(p>0.05)$ (Figure 2E). And from the GSE14764, we could conclude that higher expression of FGF9 was linked to the better prognosis of OS (HR = $0.32,95 \% \mathrm{CI}=0.11-0.9, p=0.023)$, PPS (HR $=0.34$, $95 \% \mathrm{CI}=0.11-1.03, p=0.045$ ) (Figure 2G, 2I). Additionally, OC patients with high level of FGF9 showed favorable PFS (HR $=0.72,95 \% \mathrm{CI}=0.54$ $0.97, p=0.029$ ) in GSE9891 (Figure 2H). From these results, we speculated that FGF9 could possess the potential ability to be a prognostic biomarker.

\section{Downregulated expression of FGF9 in ovarian cancer group}

Through the evaluation of the two datasets downloaded from the GEO database, we found that FGF9 expressed more highly in normal ovarian tissues than ovarian cancer tissues $(p<0.0001)$ (Figure 3A, 3B). In addition, TCGA database has verified that the expression level of FGF9 was different between the normal group and the OC group $(p=0.018)$ (Figure 3C). Meanwhile, GEPIA2.0 platform has identified that the expression level of FGF9 was higher in the normal group than that in OC group (Figure 3D). What's more, by means of the TNMplot platform, we could know about that FGF9 mRNA expression were both lower in $\mathrm{OC}$ tissues from
A

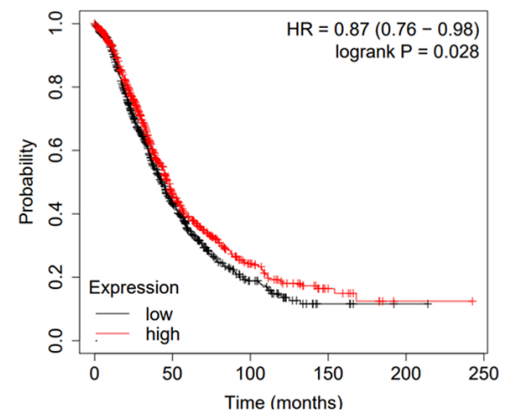

D

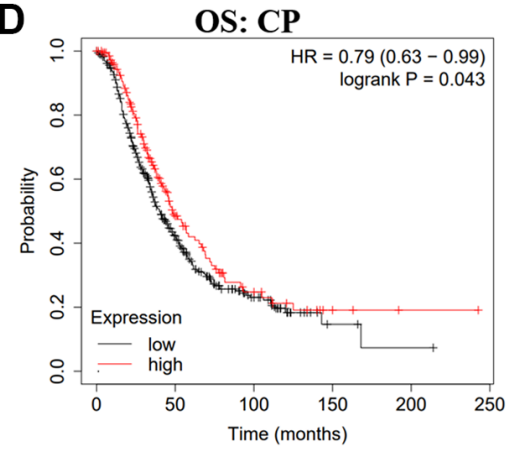

G

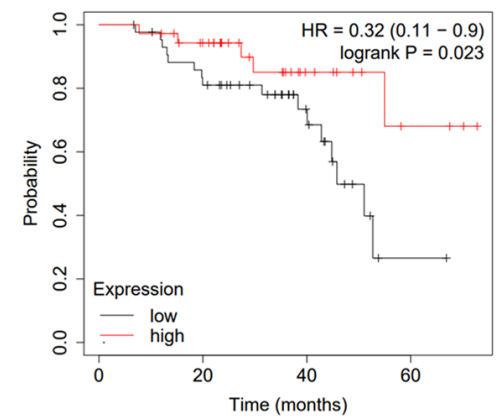

B

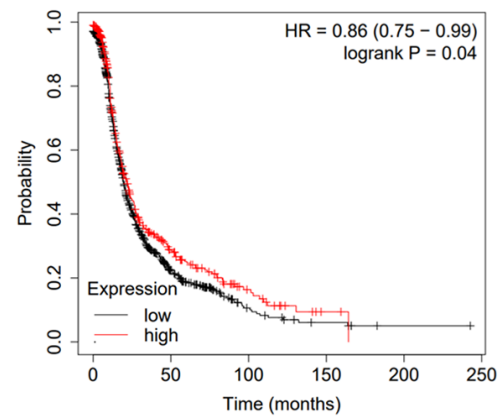

$\mathbf{E}$

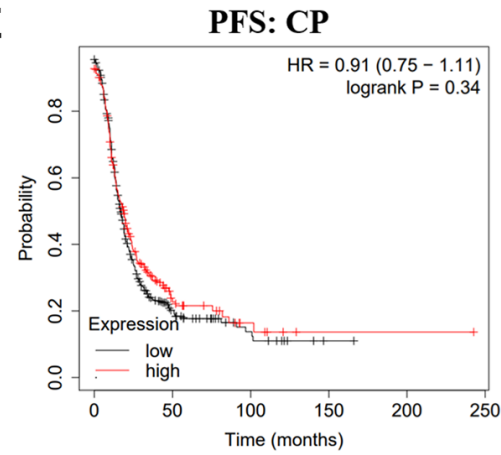

H

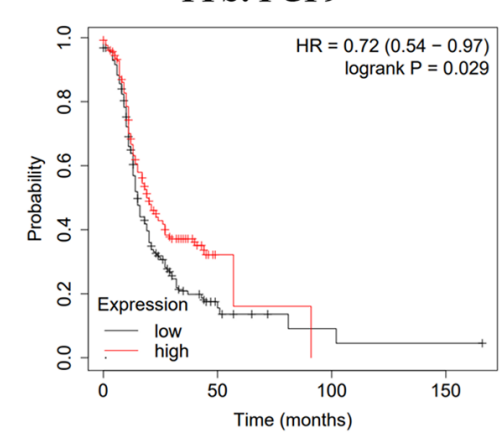

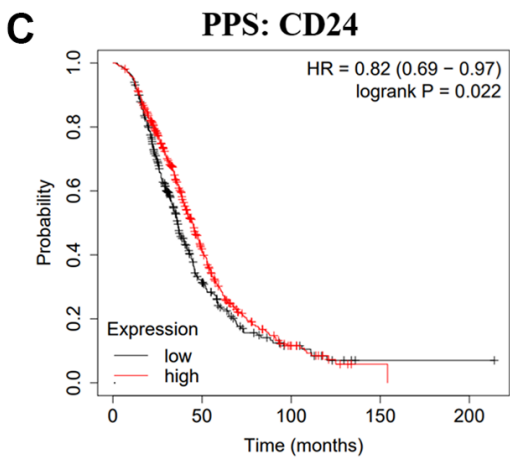

F

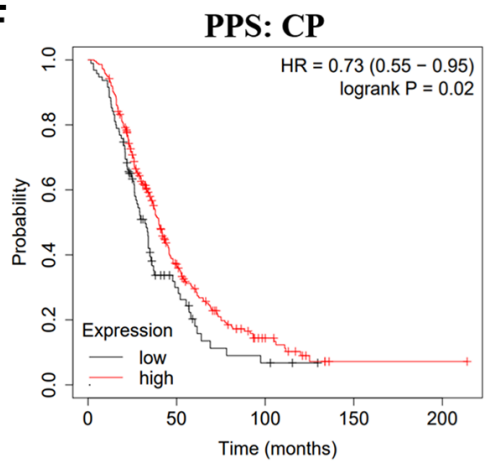

I

PPS: FGF9

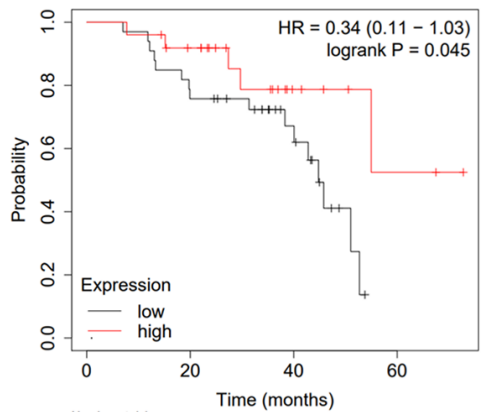

Figure 2. The prognostic values of CD24, CP and FGF9 in OC. (A-I) The prognostic values of CD24, CP and FGF9 in ovarian cancer patients. Abbreviations: OS: overall survival; PFS: progression-free survival; PPS: post progression survival. 
gene chip data $(p=1.57 \mathrm{e}-05)$ and RNA-seq data ( $p=$ 4.2e-01) (Figure 3E, 3F). The above findings have conveyed that the expression of FGF9 quietly diminished in OC group, implicating that FGF9 might be a promising inhibitor in OC patients' progression and development.

\section{The co-expression network of FGF9 in ovarian cancer}

The LinkedOmics platform was applied to explore the co-expression network in the TCGAOV cohort, and then figuring out the biological functions of FGF9 in OC patients' development. Figure 4A and Supplementary Table 2 have demonstrated the coexpressed genes that have positive and negative relationship with FGF9 $(p<0.05)$. Also, the heatmaps have depicted the genes that are positively and negatively correlated with FGF9 (Figure 4B, 4C and Supplementary Tables 3 and 4). Significantly, the top 19 positive-related genes have the great possibility to be low-risk molecules for OC patients. In addition, the top 20 negatively correlated genes that were high-risk molecules in OC. Meanwhile, 1 of top 20 negatively related genes owned the bad hazard ratio (Figure 4D). Moreover, through the investigation of Gene Ontology pathway, the result conveyed that the co-expressed genes of FGF9 participated in several biological process categories, such as response to stimulus, biological regulation and metabolic process. In the cellular component categories, these genes mainly take part in nucleus, cytosol and membrane. At the same time, the co-expressed genes of FGF9 significantly join in the protein binding, ion binding and nucleic acid binding in the molecular function categories (Figure 4E). Moreover, the KEGG analysis has implied that the enriched pathways were protein processing in endoplasmic reticulum, adipocytokine signaling pathway, epithelial cell signaling in helicobacter pylori infection, adherens junctions etc. (Figure 4F).

\section{The link between FGF9 with immune regulation}

Then, we downloaded the statistics of TCGA-OV database and analyzed the effects of FGF9 expression on immune regulation of ovarian cancer patients via ssGSEA. It indicated that FGF9 was positively linked to
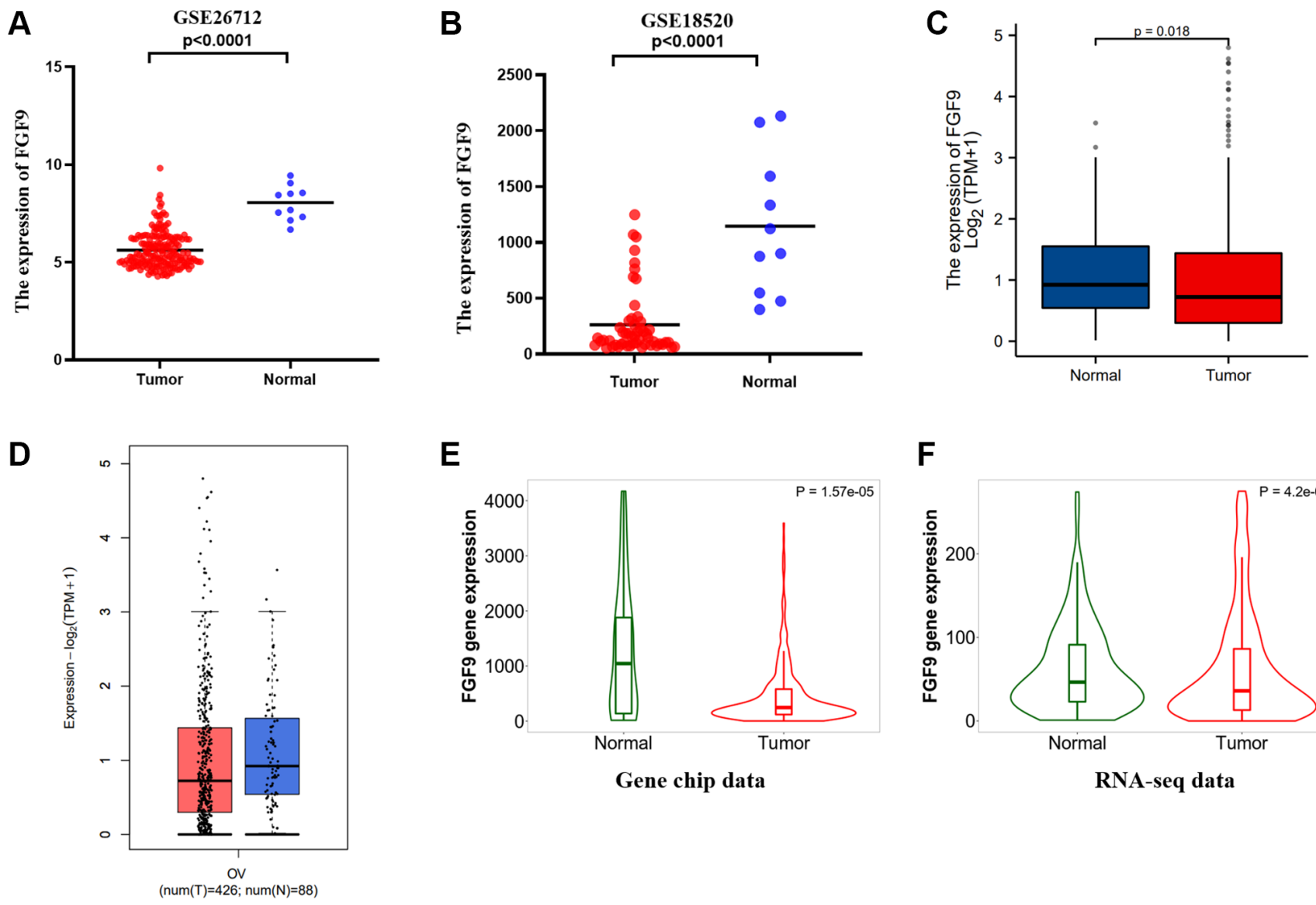

$\mathbf{E}$

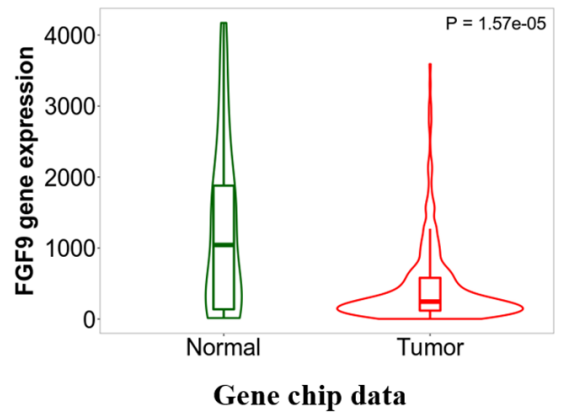

$\mathbf{F}$

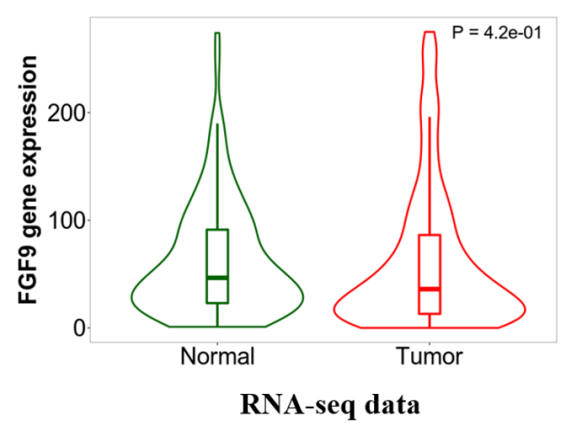

Figure 3. FGF9 was down-regulated in OC patients. (A, B) In the two datasets, the expression level of FGF9 was lower in OC tissues than that in normal ovarian tissues. (C, D) The GEPIA2.0 database and TCGA database have depicted that the expression of FGF9 decreased in OC tissues compared to normal ovarian tissues. (E, F) TNMplot database depicting FGF9 expression was lower in OC tissues compared to normal tissues from gene chip data and RNA-seq data. 
the infiltration of natural killer (NK) cells $(p<0.05)$. Meanwhile, FGF9 expression was negatively correlated with cytotoxic cells, activated dendritic cells (aDC), T cells, NK CD56dim cells, Treg, neutrophils and T helper type 1 (Th1) cells $(p<0.05)$ (Figure 5A). In addition, the results that obtained from TISIDB database were consistent with the findings before (Figure 5B). By means of TIMER database, the plot further portrayed that the expression level of FGF9 had strong negative relationship with $\mathrm{B}$ cell, $\mathrm{CD}^{+} \mathrm{T}$ cell, $\mathrm{CD}^{+} \mathrm{T}$ cell, macrophage, neutrophil and dendritic cell $(p<0.05)$ (Figure 5C). Furthermore, the figures have depicted that the relationship between FGF9 expression and immune checkpoints and the findings showed that the expression level of FGF9 was negatively linked with CTLA4 (Spearman $\mathrm{r}=-0.239, p<0.001$ ) and VSIR (Spearman $r=-0.169,-<0.001$ ) (Figure 5D, 5E).

Additionally, we further investigated the relation between FGF9 and immune responses through the TISIDB platform, including immunoinhibitors and receptors. This picture has conveyed the correlation between immunoinhibitors in $\mathrm{OC}$ and the expression of
FGF9 (Supplementary Figure 1A). The findings implicated that the top four immunoinhibitors that were strongly linked with FGF9 were PDCD1LG2 (Spearman $\mathrm{r}=-0.331, p=3.36 \mathrm{e}-09$ ), HAVCR2 (Spearman $\mathrm{r}=-0.317, p=1.66 \mathrm{e}-08), \quad \mathrm{CD} 274$ (Spearman $\mathrm{r}=-0.314, p=2.19 \mathrm{e}-08)$ and LAG3 (Spearman $\mathrm{r}=-0.309, p=3.96 \mathrm{e}-08$ ) (Supplementary Figure 1B). Moreover, the correlation between FGF9 and receptors has been conducted (Supplementary Figure 2A). And the top four receptors that had negative association with FGF9 expression were CXCR6 (Spearman $\mathrm{r}=-0.289, p=2.96 \mathrm{e}-07$ ), CXCR3 (Spearman $\mathrm{r}=-0.271, p=1.52 \mathrm{e}-06)$, CCR5 (Spearman $\mathrm{r}=-0.264, p=3.04 \mathrm{e}-06$ ) and CCR1 (Spearman $\mathrm{r}=$ $-0.259, p=4.7 \mathrm{e}-06$ ) (Supplementary Figure 2B). In summary, these results have demonstrated that FGF9 was involved in the immune regulation of OC patients.

\section{DISCUSSION}

The purpose of this paper was to figure out the correlation of exosome-associated genes and the progression in ovarian cancer. By exploring the
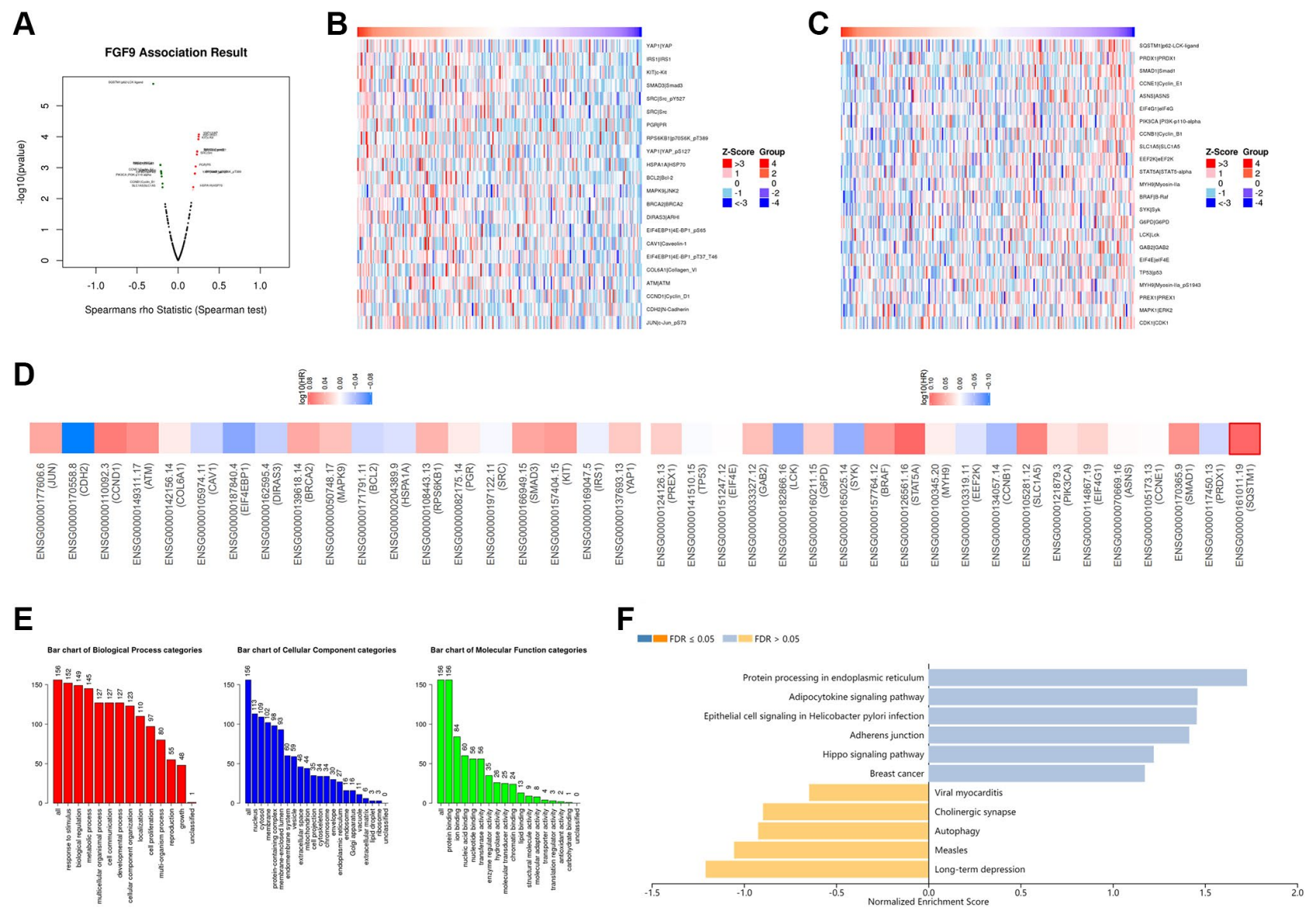

Figure 4. The co-expression network of FGF9 in OC. (A) The LinkedOmics platform portraying the crucially associated genes with FGF9 in OC patients. (B, C) Heatmaps showing the top genes that were positively and negatively correlated with FGF9 in OC. (D) Survival heatmaps downloaded from the GEPIA2.0 database displayed that the top genes that were positively and negatively associated with FGF9 in OC. (E, F) GO signaling pathway and KEGG signaling pathway of FGF9 in OC patients. 
co-DEGs between an exosome-associated gene dataset and two OC datasets obtained from the GEO database, we could find two up-regulated genes CD24 and CP. And we concluded the expression level of FGF9 displayed downregulation of OC. Additionally, the higher expression of FGF9 tended to be correlated with
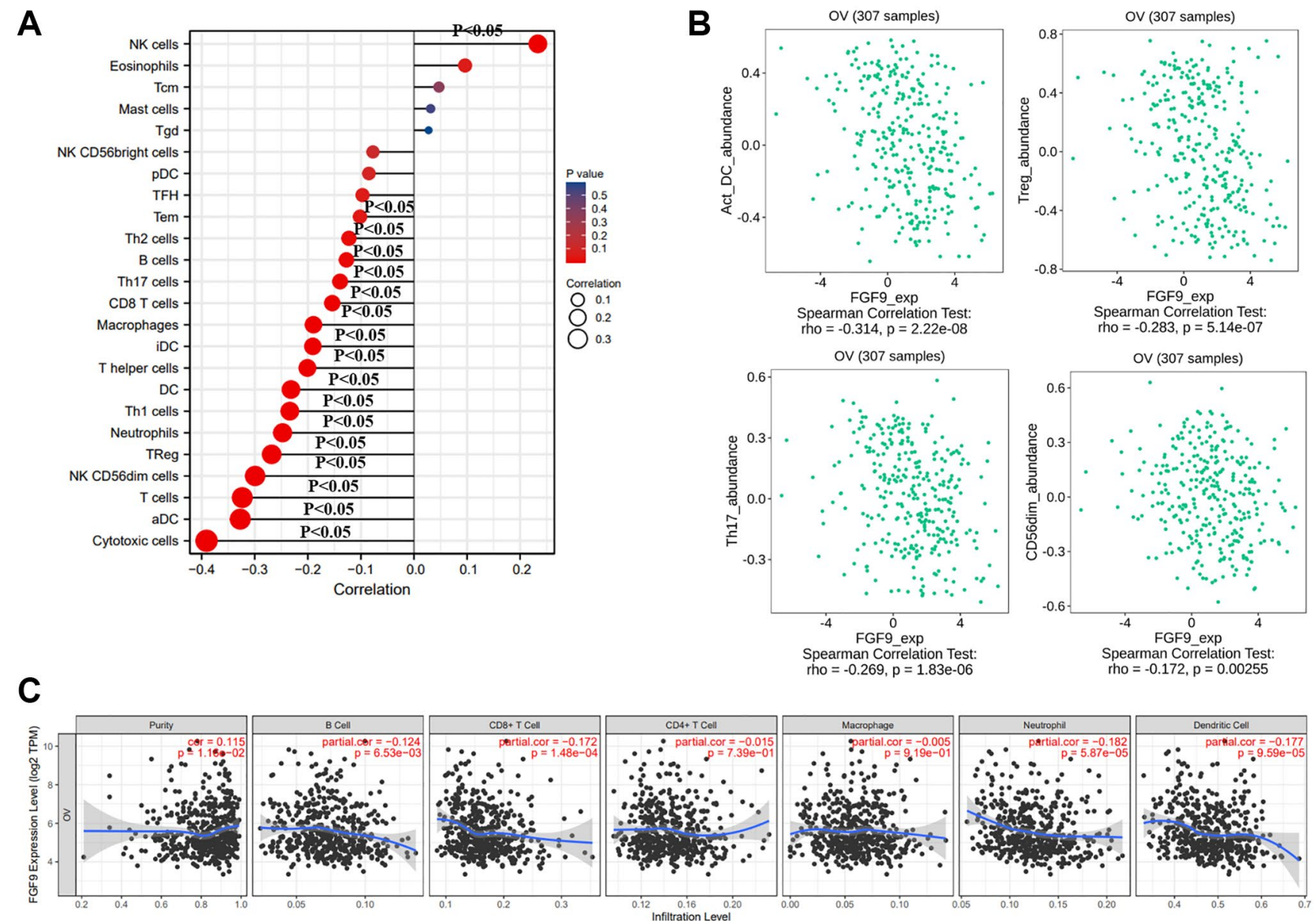
FGF9 expression decreased in OC group. By means of LinkedOmics database, we could figure out the positively and negatively associated genes with FGF9 in
D

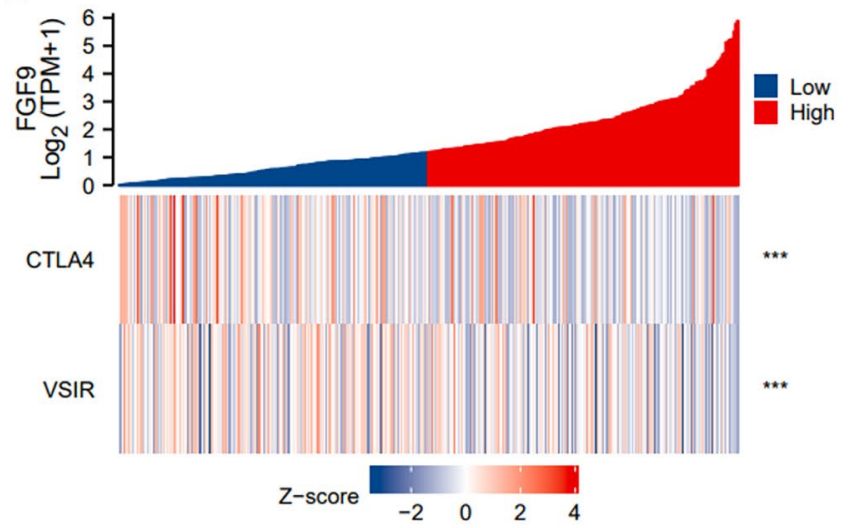

E

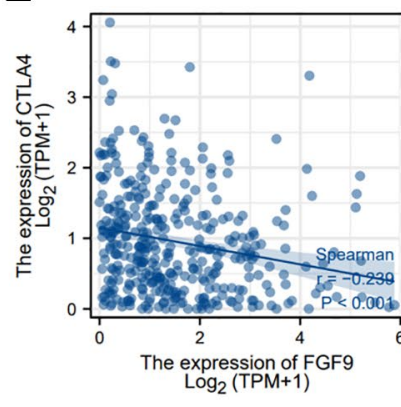

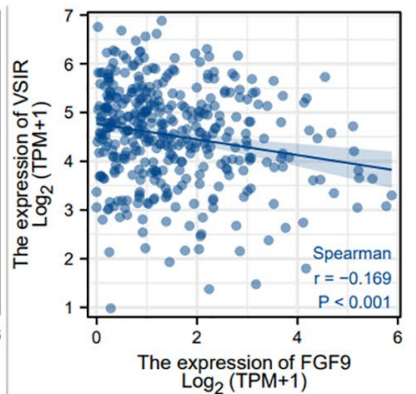

Figure 5. The relationship between the expression level of FGF9 and immune responses of OC patients. (A) The diagraph showing the relation between FGF9 expression and 24 types of immune cells. The size of the dots represented the values of Spearman $r$ $(p<0.05)$. (B) The pictures downloaded from TISIDB database showing the relationship between FGF9 and immune infiltration cells, such as activated dendritic cells (aDC), Treg, Th17 cells, NK CD56dim cells $(p<0.05)$. (C) The Timer database showing the relationship between the expression level of FGF9 and immune infiltration cells. (D, E) The heatmap and scatterplot depicting FGF9 expression was negatively correlated to VSIR or CTLA4. 
OC patients. Meanwhile, FGF9 showed a correlation with the prognosis of OC patients. Also, the results of GO pathway and KEGG pathway conveyed that the co-expressed genes participated in microenvironment of cancer cells and the biological functions in treating cancers.

It has been reported that various kinds of cells could generate exosomes. And the diameters of exosomes are 30-150 $\mathrm{nm}$. Moreover, exosomes have been identified to play an essential role in the cells' communication via miRNA, mRNA, and DNA [25]. Recently, there is an increasing trend concerning the exosome nanotechnology [26]. Because exosome possessed the special structure and favorable biological characteristic, the studies regarding exosome could be beneficial to the nano medical therapy in the future [27]. Additionally, exosomes could be secreted by tumor cells and they could exert great effects in changing the microenvironment of tumors. At the same time, it was verified that exosomes were significant in the cancer progression and the antitumor immunity [28, 29]. Several signaling pathways could be induced and suppressed by exosomes. Moreover, exosomes could contribute to the metastasis and drug resistance of cancer cells [30]. Significantly, exosomes that were generated from OC cells could induce niche formatting before metastasis through inhibiting immune responses, angiogenesis and the reformation of oncogenes [31]. A recent study has indicated that, in OC cells, the exosomal miR-1246 induce the development of tumors. Besides, the inhibitors of miR-1246 could lead to the downregulated level of PDGFR $\beta$ and ki67 in vivo trials. Also, this paper has demonstrated that miR-1246 secretion through exosomes could elevate the chemoresistance in OC patients [32]. In addition, another study has illustrated that exosomal pGSN could elevate the survival of OC cells through the conversion of the chemosensitivity into chemoresistance in OC cells [33]. These results have implicated that in OC patients' progression, exosome was importantly vital. And to further investigate the underlying mechanism of exosome and $\mathrm{OC}$ could benefit the future therapies for OC patients. Our study is aimed at exploring the prognostic value of FGF9 in OC patients and we concluded that high expression level of FGF9 was followed with good prognosis.

FGF9, as a target gene of miR-214, could suppress cancer-associated fibroblasts (CAFs) in GC cells. Also, the abnormal FGF9 expression in CAFs has strong relationship with poorer prognosis of GC patients [34]. A study implicated that HD5 expressed in ovarian cancer cells via western blot and immunohistochemistry (IHC) evaluations. And the expression of HD5 mediated by FGF9 signaling pathway further verified HD5 was correlated with cancer [35]. The analysis has conveyed that overexpression of FGF9 could provide a new clinical strategy in gastric cancer and bladder cancer. P4, as a new FGF9-binding peptide, could play a pivotal part in enhancing the sensitivity of chemical drugs [36]. Low expression of miR-187 was linked to diminished overall survival (OS) of cervical cancer patients. The over-expressed level of miR-187 suppressed cell growth and triggered cell apoptosis in cervical cancer [37]. FGF9 could act as a target gene of microRNA-219a-5p and relieved the chemoresistance of cisplatin in nonsmall cell lung cancer (NSCLC) [38]. In this paper, the exosome-related gene FGF9 expression was high in normal ovarian tissues compared to OC tissues.

Several studies have proven that the combination of immunotherapy and radiotherapy could provide a new strategy of therapy in ovarian cancer patients. MSIH/dMMR and immune checkpoint inhibition could synergize with other strategies to exert effects in the treatment of ovarian cancer. Cancer vaccines and immunomodulation were reported to possess the potential to be low-toxicity approaches for OC patients [39-41]. In this article, we have explored the correlation between FGF9 and immune responses. FGF9 was reported to have a positive link with NK cells. On the other hand, FGF9 had a negative correlation with cytotoxic cells, aDC, T cells, NK CD56dim cells, Treg, neutrophils and Th1 cells. Simultaneously, FGF9 was crucially related to immunoinhibitors (PDCD1LG2, HAVCR2, CD274, LAG3) and receptors (CXCR6, CXCR3, CCR5, CCR1). Moreover, cancers might evade from the attack of NK cells. Interestingly, to reinforce the cytotoxicity of NK cells could elevate the efficacy of NK-associated immunotherapy [42]. Also, in high-grade serous ovarian cancer (HGSC), NK cells could be pivotal in the anticancer immunity triggered by immune checkpoint blockade [43]. This study also concluded that FGF9 expression had a negative relation with immune checkpoints, including CTLA4 and VSIR. CTLA4 belonged to the family of Immunoglobulinassociated receptors and it was expressed via $\mathrm{CD} 4^{+}$and $\mathrm{CD}^{+} \mathrm{T}$ cells. Through this way, CTLA4 could take part in activating the $\mathrm{T}$ cells and induce immune regulation [44]. Nowadays, immune checkpoint inhibitors were applied for the therapy of ovarian cancer. CTLA4 could exert great effects in eliminating autoreactive $\mathrm{T}$ cells and PD-1 controlled T cells' apoptosis [45]. These above results have implicated that FGF9 was correlated with immune responses and regulation, implying that FGF9 could be a promising biomarker for the immunotherapy of $\mathrm{OC}$ patients.

\section{CONCLUSIONS}

In conclusion, this paper has reported that the expression level of FGF9 had a vital relationship with 
the prognostic prediction value of OC. Furthermore, it has been identified that FGF9 was crucially related with the immunoinhibitors and chemokine receptors. Consequently, this study has provided a novel horizon that the exosome-correlated gene FGF9 had the great potential to be a prognostic prediction strategy for OC patients.

\section{AUTHOR CONTRIBUTIONS}

Xu Z, Cai Y and Yan Y: acquisition of data. Cai Y, Liu W, Kang F, He Q and Peng J: analysis and interpretation of data. Hong Q, Zhang $\mathrm{W}$ and Li J: conception and design. Cai Y and Yan Y: data curation. Liu W: development of methodology. Xu Z and Cai Y: writing the manuscript and revision of the manuscript. All authors contributed to the article and approved the submitted version.

\section{CONFLICTS OF INTEREST}

The authors declare no conflicts of interest related to this study.

\section{FUNDING}

This study is supported by grants from the Science and Technology Innovation Program of Hunan Province (2021RC3029), the Natural Science Foundation of Hunan Province (2021JJ30904, 2020JJ5934), the horizontal project $(1$ 43010100) and the China Postdoctoral Science Foundation (2021T140754, 2020M672521).

\section{REFERENCES}

1. Cai Y, Wu G, Peng B, Li J, Zeng S, Yan Y, Xu Z. Expression and molecular profiles of the AlkB family in ovarian serous carcinoma. Aging (Albany NY). 2021; 13:9679-92.

https://doi.org/10.18632/aging.202716 PMID:33744868

2. Odunsi K. Immunotherapy in ovarian cancer. Ann Oncol. 2017 (suppl 8); 28:viii1-7. https://doi.org/10.1093/annonc/mdx444 PMID:29232467

3. Xu Z, Wang X, Zeng S, Ren X, Yan Y, Gong Z. Applying artificial intelligence for cancer immunotherapy. Acta Pharm Sin B. 2021; 11:3393-405.

https://doi.org/10.1016/i.apsb.2021.02.007 PMID: $\underline{34900525}$

4. Deng D, Luo X, Zhang S, Xu Z. Immune cell infiltrationassociated signature in colon cancer and its prognostic implications. Aging (Albany NY). 2021; 13:19696-709. https://doi.org/10.18632/aging.203380 PMID: $\underline{34349038}$

5. Drakes ML, Stiff PJ. Regulation of Ovarian Cancer Prognosis by Immune Cells in the Tumor Microenvironment. Cancers (Basel). 2018; 10:302. https://doi.org/10.3390/cancers10090302 PMID: 30200478

6. Thakur A, Parra DC, Motallebnejad P, Brocchi M, Chen HJ. Exosomes: Small vesicles with big roles in cancer, vaccine development, and therapeutics. Bioact Mater. 2022; 10:281-94. https://doi.org/10.1016/i.bioactmat.2021.08.029 PMID:34901546

7. Thakur A, Ke X, Chen YW, Motallebnejad P, Zhang K, Lian $Q$, Chen HJ. The mini player with diverse functions: extracellular vesicles incell biology, disease, and therapeutics. Protein Cell. 2021. [Epub ahead of print]. https://doi.org/10.1007/s13238-021-00863-6 PMID:34374936

8. Xu Z, Zeng S, Gong Z, Yan Y. Exosome-based immunotherapy: a promising approach for cancer treatment. Mol Cancer. 2020; 19:160. https://doi.org/10.1186/s12943-020-01278-3 PMID:33183286

9. Thakur A, Sidu RK, Zou H, Alam MK, Yang M, Lee Y. Inhibition of Glioma Cells' Proliferation by Doxorubicin-Loaded Exosomes via Microfluidics. Int J Nanomedicine. 2020; 15:8331-43.

https://doi.org/10.2147/IJN.S263956

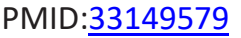

10. Peng J, Liang Q, Xu Z, Cai Y, Peng B, Li J, Zhang W, Kang $F$, Hong $Q$, Yan $Y$, Zhang $M$. Current Understanding of Exosomal MicroRNAs in Glioma Immune Regulation and Therapeutic Responses. Front Immunol. 2022; 12:813747. https://doi.org/10.3389/fimmu.2021.813747 PMID: $\underline{3095909}$

11. Huang CW, Lu SY, Huang TC, Huang BM, Sun HS, Yang SH, Chuang JI, Hsueh YY, Wu YT, Wu CC. FGF9 induces functional differentiation to Schwann cells from human adipose derived stem cells. Theranostics. 2020; 10:2817-31.

https://doi.org/10.7150/thno.38553

PMID:32194837

12. Ishioka K, Yasuda H, Hamamoto J, Terai H, Emoto K, Kim TJ, Hirose S, Kamatani T, Mimaki S, Arai D, Ohgino K, Tani T, Masuzawa K, et al. Upregulation of FGF9 in Lung Adenocarcinoma Transdifferentiation to Small Cell Lung Cancer. Cancer Res. 2021; 81:3916-29. https://doi.org/10.1158/0008-5472.CAN-20-4048 PMID:34083250 
13. Bhattacharya R, Ray Chaudhuri S, Roy SS. FGF9induced ovarian cancer cell invasion involves VEGFA/VEGFR2 augmentation by virtue of ETS1 upregulation and metabolic reprogramming. J Cell Biochem. 2018; 119:8174-89.

https://doi.org/10.1002/jcb.26820

PMID:29904943

14. Clough E, Barrett T. The Gene Expression Omnibus Database. Methods Mol Biol. 2016; 1418:93-110. https://doi.org/10.1007/978-1-4939-3578-9 5 PMID:27008011

15. Bonome T, Levine DA, Shih J, Randonovich M, PiseMasison CA, Bogomolniy F, Ozbun L, Brady J, Barrett JC, Boyd J, Birrer MJ. A gene signature predicting for survival in suboptimally debulked patients with ovarian cancer. Cancer Res. 2008; 68:5478-86. https://doi.org/10.1158/0008-5472.CAN-07-6595 PMID:18593951

16. Vathipadiekal V, Wang V, Wei W, Waldron L, Drapkin R, Gillette M, Skates S, Birrer M. Creation of a Human Secretome: A Novel Composite Library of Human Secreted Proteins: Validation Using Ovarian Cancer Gene Expression Data and a Virtual Secretome Array. Clin Cancer Res. 2015; 21:4960-9. https://doi.org/10.1158/1078-0432.CCR-14-3173 PMID:25944803

17. Mok SC, Bonome T, Vathipadiekal V, Bell A, Johnson ME, Wong KK, Park DC, Hao K, Yip DK, Donninger $\mathrm{H}$, Ozbun L, Samimi G, Brady J, et al. A gene signature predictive for outcome in advanced ovarian cancer identifies a survival factor: microfibril-associated glycoprotein 2. Cancer Cell. 2009; 16:521-32.

https://doi.org/10.1016/j.ccr.2009.10.018 PMID:19962670

18. McCain J. The cancer genome atlas: new weapon in old war? Biotechnol Healthc. 2006; 3:46-51B.

PMID:23424349

19. Lánczky A, Győrffy B. Web-Based Survival Analysis Tool Tailored for Medical Research (KMplot): Development and Implementation. J Med Internet Res. 2021; 23:e27633.

https://doi.org/10.2196/27633

PMID:34309564

20. Bartha Á, Győrffy B. TNMplot.com: A Web Tool for the Comparison of Gene Expression in Normal, Tumor and Metastatic Tissues. Int J Mol Sci. 2021; 22:2622. https://doi.org/10.3390/ijms22052622 PMID:33807717

21. Tang Z, Kang B, Li C, Chen T, Zhang Z. GEPIA2: an enhanced web server for large-scale expression profiling and interactive analysis. Nucleic Acids Res. 2019; 47:W556-60. https://doi.org/10.1093/nar/gkz430

PMID:31114875

22. Vasaikar SV, Straub P, Wang J, Zhang B. LinkedOmics: analyzing multi-omics data within and across 32 cancer types. Nucleic Acids Res. 2018; 46:D956-63. https://doi.org/10.1093/nar/gkx1090 PMID:29136207

23. Ru B, Wong CN, Tong $\mathrm{Y}$, Zhong JY, Zhong SSW, Wu WC, Chu KC, Wong CY, Lau CY, Chen I, Chan NW, Zhang J. TISIDB: an integrated repository portal for tumor-immune system interactions. Bioinformatics. 2019; 35:4200-2.

https://doi.org/10.1093/bioinformatics/btz210 PMID:30903160

24. Li T, Fan J, Wang B, Traugh N, Chen Q, Liu JS, Li B, Liu XS. TIMER: A Web Server for Comprehensive Analysis of Tumor-Infiltrating Immune Cells. Cancer Res. 2017; 77:e108-10.

https://doi.org/10.1158/0008-5472.CAN-17-0307 PMID:29092952

25. Yang $D$, Zhang $W$, Zhang $H$, Zhang $F$, Chen $L$, Ma $L$, Larcher LM, Chen S, Liu N, Zhao Q, Tran PHL, Chen C, Veedu RN, Wang T. Progress, opportunity, and perspective on exosome isolation - efforts for efficient exosome-based theranostics. Theranostics. 2020; 10:3684-707.

https://doi.org/10.7150/thno.41580

PMID: $\underline{32206116}$

26. Thakur A, Qiu G, Ng SP, Guan J, Yue J, Lee Y, Wu CL. Direct detection of two different tumor-derived extracellular vesicles by SAM-AuNIs LSPR biosensor. Biosens Bioelectron. 2017; 94:400-7. https://doi.org/10.1016/j.bios.2017.03.036 PMID:28324860

27. Yang B, Chen Y, Shi J. Nanocatalytic Medicine. Adv Mater. 2019; 31:e1901778. https://doi.org/10.1002/adma.201901778 PMID: $\underline{31328844}$

28. Zhang L, Yu D. Exosomes in cancer development, metastasis, and immunity. Biochim Biophys Acta Rev Cancer. 2019; 1871:455-68. https://doi.org/10.1016/j.bbcan.2019.04.004 PMID:31047959

29. Thakur A, Qiu G, Xu C, Han X, Yang T, Ng SP, Chan KWY, Wu CML, Lee Y. Label-free sensing of exosomal MCT1 and CD147 for tracking metabolic reprogramming and malignant progression in glioma. Sci Adv. 2020; 6:eaaz6119. https://doi.org/10.1126/sciadv.aaz6119 PMID:32637597

30. Zhong $Y$, Li H, Li P, Chen Y, Zhang M, Yuan Z, Zhang $Y$, Xu Z, Luo G, Fang Y, Li X. Exosomes: A New Pathway 
for Cancer Drug Resistance. Front Oncol. 2021; 11:743556.

https://doi.org/10.3389/fonc.2021.743556

PMID:34631581

31. Feng W, Dean DC, Hornicek FJ, Shi H, Duan Z. Exosomes promote pre-metastatic niche formation in ovarian cancer. Mol Cancer. 2019; 18:124. https://doi.org/10.1186/s12943-019-1049-4 PMID:31409361

32. Kanlikilicer $\mathrm{P}$, Bayraktar R, Denizli $\mathrm{M}$, Rashed $\mathrm{MH}$, Ivan C, Aslan B, Mitra R, Karagoz K, Bayraktar E, Zhang X, Rodriguez-Aguayo C, El-Arabey AA, Kahraman N, et al. Exosomal miRNA confers chemo resistance via targeting Cav1/p-gp/M2-type macrophage axis in ovarian cancer. EBioMedicine. 2018; 38:100-12. https://doi.org/10.1016/i.ebiom.2018.11.004 PMID:30487062

33. Asare-Werehene M, Nakka K, Reunov A, Chiu CT, Lee WT, Abedini MR, Wang PW, Shieh DB, Dilworth FJ, Carmona E, Le T, Mes-Masson AM, Burger D, Tsang BK. The exosome-mediated autocrine and paracrine actions of plasma gelsolin in ovarian cancer chemoresistance. Oncogene. 2020; 39:1600-16. https://doi.org/10.1038/s41388-019-1087-9 PMID:31700155

34. Wang R, Sun Y, Yu W, Yan Y, Qiao M, Jiang R, Guan W, Wang L. Downregulation of miRNA-214 in cancerassociated fibroblasts contributes to migration and invasion of gastric cancer cells through targeting FGF9 and inducing EMT. J Exp Clin Cancer Res. 2019; 38:20. https://doi.org/10.1186/s13046-018-0995-9 PMID:30646925

35. Vragniau C, Hübner JM, Beidler $\mathrm{P}$, Gil S, Saydaminova K, Lu ZZ, Yumul R, Wang H, Richter M, Sova P, Drescher C, Fender $\mathrm{P}$, Lieber A. Studies on the Interaction of Tumor-Derived HD5 Alpha Defensins with Adenoviruses and Implications for Oncolytic Adenovirus Therapy. J Virol. 2017; 91:e02030-16.

https://doi.org/10.1128/JVI.02030-16 PMID:28077642

36. Wang J, Tan X, Guo Q, Lin X, Huang Y, Chen L, Zeng X, Li R, Wang $H$, Wu X. FGF9 inhibition by a novel binding peptide has efficacy in gastric and bladder cancer per se and reverses resistance to cisplatin. Pharmacol Res. 2020; 152:104575.

https://doi.org/10.1016/i.phrs.2019.104575 PMID: $\underline{31805343}$

37. Liang H, Luo R, Chen X, Zhao Y, Tan A. miR-187 inhibits the growth of cervical cancer cells by targeting FGF9. Oncol Rep. 2017; 38:1977-84.

https://doi.org/10.3892/or.2017.5916

PMID:28849071
38. Rao C, Miao X, Zhao G, Zhang C, Shen H, Dong C, Yang M. MiR-219a-5p enhances cisplatin sensitivity of human non-small cell lung cancer by targeting FGF9. Biomed Pharmacother. 2019; 114:108662. https://doi.org/10.1016/i.biopha.2019.108662 PMID:30999114

39. Herrera FG, Irving M, Kandalaft LE, Coukos G. Rational combinations of immunotherapy with radiotherapy in ovarian cancer. Lancet Oncol. 2019; 20:e417-33. https://doi.org/10.1016/S1470-2045(19)30401-2 PMID:31364594

40. Morand S, Devanaboyina M, Staats $H$, Stanbery $L$, Nemunaitis J. Ovarian Cancer Immunotherapy and Personalized Medicine. Int J Mol Sci. 2021; 22:6532. https://doi.org/10.3390/ijms22126532 PMID: $\underline{34207103}$

41. Kandalaft LE, Odunsi K, Coukos G. Immunotherapy in Ovarian Cancer: Are We There Yet? J Clin Oncol. 2019; 37:2460-71. https://doi.org/10.1200/JCO.19.00508 PMID:31403857

42. Pomeroy EJ, Hunzeker JT, Kluesner MG, Lahr WS, Smeester BA, Crosby MR, Lonetree CL, Yamamoto K, Bendzick L, Miller JS, Geller MA, Walcheck B, Felices $M$, et al. A Genetically Engineered Primary Human Natural Killer Cell Platform for Cancer Immunotherapy. Mol Ther. 2020; 28:52-63. https://doi.org/10.1016/j.ymthe.2019.10.009 PMID: $\underline{31704085}$

43. Wan C, Keany MP, Dong $\mathrm{H}$, Al-Alem LF, Pandya UM, Lazo S, Boehnke K, Lynch KN, Xu R, Zarrella DT, Gu S, Cejas P, Lim K, et al. Enhanced Efficacy of Simultaneous PD-1 and PD-L1 Immune Checkpoint Blockade in High-Grade Serous Ovarian Cancer. Cancer Res. 2021; 81:158-73.

https://doi.org/10.1158/0008-5472.CAN-20-1674 PMID:33158814

44. Rowshanravan B, Halliday N, Sansom DM. CTLA-4: a moving target in immunotherapy. Blood. 2018; 131:58-67.

https://doi.org/10.1182/blood-2017-06-741033 PMID:29118008

45. Fan CA, Reader J, Roque DM. Review of Immune Therapies Targeting Ovarian Cancer. Curr Treat Options Oncol. 2018; 19:74. https://doi.org/10.1007/s11864-018-0584-3 PMID: $\underline{30430276}$ 


\section{SUPPLEMENTARY MATERIALS}

\section{Supplementary Figures}
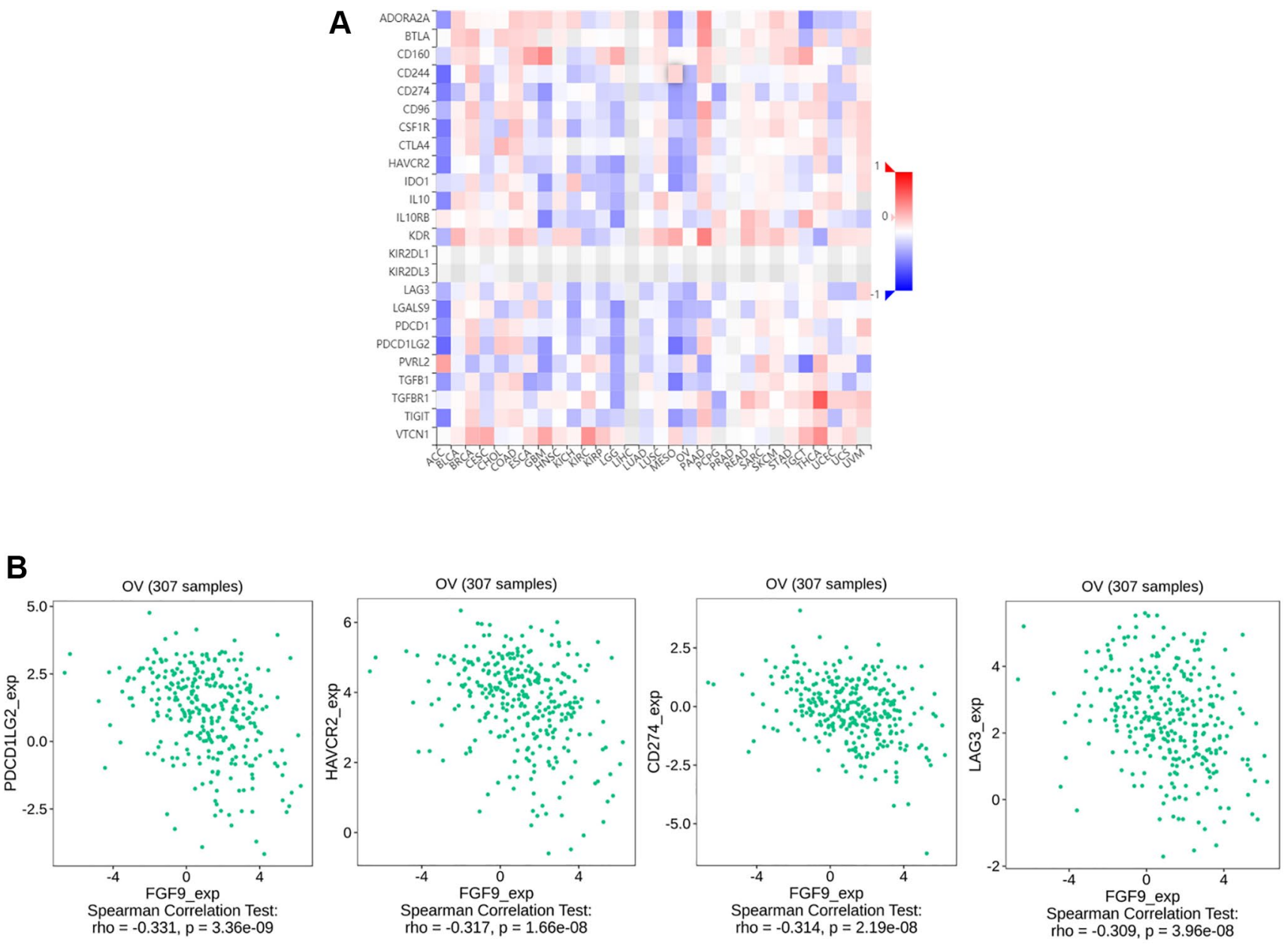

Supplementary Figure 1. The relationship between the expression level of FGF9 and immunoinhibitors of OC patients.

(A) The diagraph showing the correlation between FGF9 expression and immunoinhibitors. (B) The scatter plots depicting the top four immunoinhibitors sharing a negative relationship with FGF9 expression. 

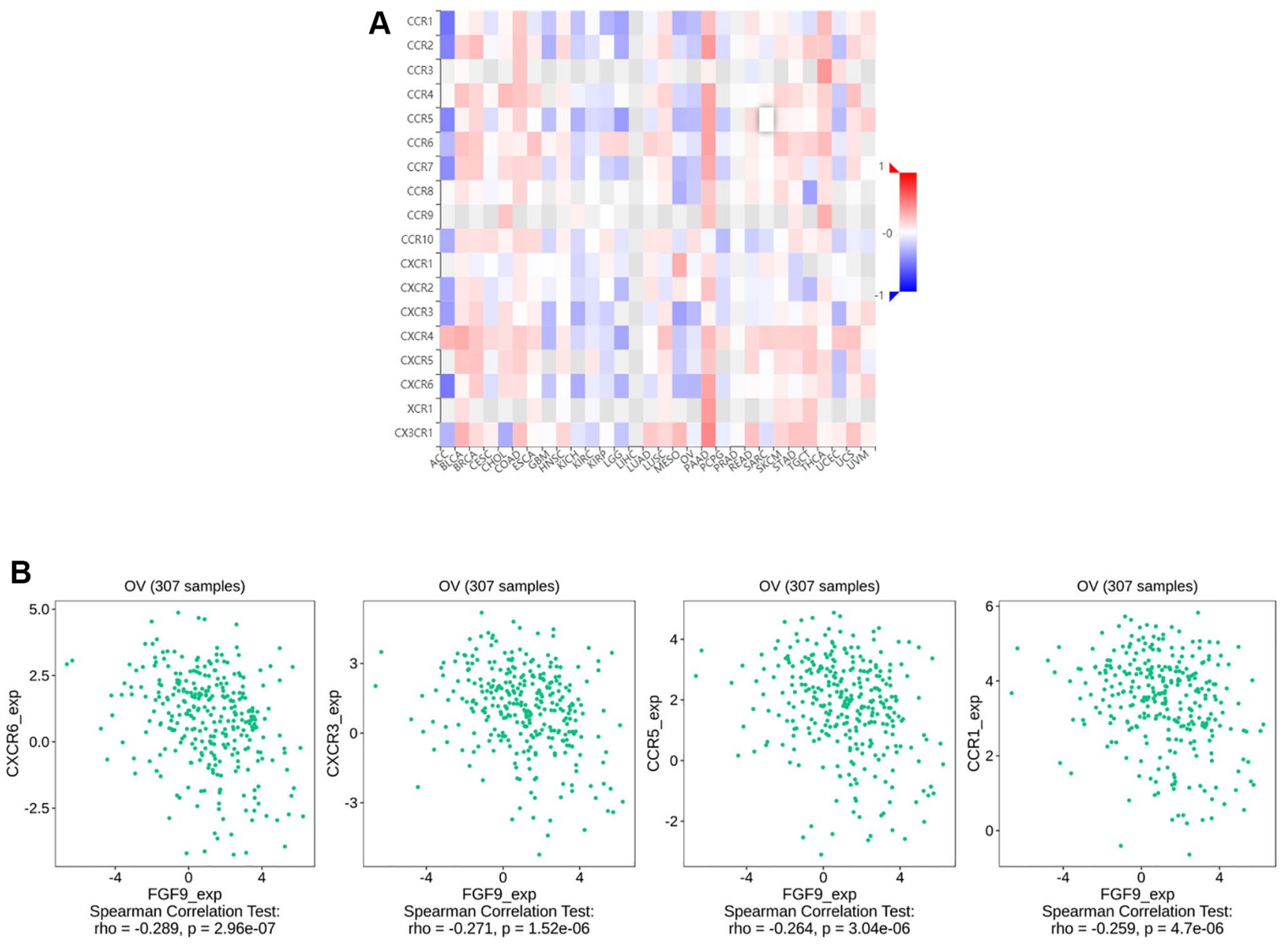

Supplementary Figure 2. The correlation between the FGF9 expression and chemokine receptors of OC patients. (A) The picture showing the connection between FGF9 expression and receptors. (B) The scatter plots portraying the top four receptors that possessed a negative relationship with FGF9 expression. 


\section{Supplementary Tables}

Please browse Full Text version to see the data of Supplementary Tables 1 and 2.

Supplementary Table 1. The upregulated genes and downregulated genes in the two GEO datasets.

Supplementary Table 2. The co-expressed genes possessing positive and negative relationship with FGF9.

Supplementary Table 3. The top 19 genes positively correlated with FGF9 in ovarian cancer.

\begin{tabular}{cccc}
\hline YAP1 & PGR & BRCA2 & ATM \\
IRS1 & RPS6KB1 & DIRAS3 & CCND1 \\
KIT & HSPA1A & EIF4EBP1 & CDH2 \\
SMAD3 & BCL2 & CAV1 & JUN \\
SRC & MAPK9 & COL6A1 & \\
\hline
\end{tabular}

Supplementary Table 4 . The top 20 genes negatively correlated with FGF9 in ovarian cancer.

\begin{tabular}{cccc}
\hline SQSTM1 & EIF4G1 & MYH9 & LCK \\
PRDX1 & PIK3CA & STAT5A & GAB2 \\
SMAD1 & SLC1A5 & BRAF & EIF4E \\
CCNE1 & CCNB1 & SYK & TP53 \\
ASNS & GEF2K & G6P & PREX1 \\
\hline
\end{tabular}

